Int. J. Dev. Biol. 61: 165-170 (2017)

doi: $10.1387 / \mathrm{ijdb} .160271 \mathrm{ht}$

\title{
microRNAs in Drosophila regulate cell fate by repressing single mRNA targets
}

\author{
NOAM PERRY, MARINA VOLIN and HILA TOLEDANO* \\ Department of Human Biology, Faculty of Natural Sciences, University of Haifa, Haifa, Israel
}

\begin{abstract}
Regulation of gene expression governs all aspects of the lifespan of the organism, such as embryonic development, stem cell differentiation, reproduction and aging. Among the most important regulators of these extremely complex processes are microRNAs (miRNAs), small non-coding RNAs that repress gene expression by binding to primary sequences on the mRNA of their target. Theoretically, the mere existence of a miRNA recognition sequence on a given mRNA is sufficient to generate a functional response. Since these short sequences are abundant, one miRNA can potentially bind to multiple targets, thus generating endless possible biological outcomes. However, is this really the case? Bioinformatics and molecular biology tools provide theoretical interaction predictions, but the data obtained by these methods is often too general and is impaired by false identifications. Therefore, a better understanding of the biological role of miRNAs requires mapping of the exact miRNA-mRNA interactions that occur in vivo. Drosophila melanogaster provides several unique advantages over other model organisms in the study of miRNA functional targeting. The majority of its miRNAs are evolutionarily conserved up to humans, suggesting that they regulate similar pathways across organisms. Complete genome-wide collections make Drosophila the only organism that enables constitutive and inducible gain and loss-of function manipulations of all annotated miRNAs. These powerful tools led to several groundbreaking discoveries of the role that miRNAs play in regulation of development, stem-cell function and aging, and proved that although many outcomes are possible, most Drosophila miRNAs regulate a single phenotype through downregulation of a single major mRNA target.
\end{abstract}

KEY WORDS: microRNAs, Drosophila, miRNA-sponge, miRNA-sensor, mRNA targets

\section{Introduction}

As their name implies, microRNAs (miRNAs) are small RNA molecules ranging between 18-26 nucleotides. Like messenger RNAs (mRNAs), miRNAs are encoded in the organism genome of eukaryotic cells, transcribed by RNA polymerase II, processed at the nucleus and exported to the cytoplasm. At the cytoplasm, their function is to uncouple targeted mRNAs from the translation machinery and thus prevent their translation into active proteins (Carthew et al., 2016, Ghildiyal and Zamore, 2009). Fortunately, the answer for the intriguing question of why should the cell transcribe an mRNA, process it and export it into the cytoplasm only to be degraded by a miRNA, came immediately with the discovery of miRNAs in $C$. elegans. The function of the first miRNAs identified, lin-14 and let-7(Lee and Ambros, 2001, Lee et al., 1993, Wightman etal., 1993), is to clear out the transcription program that defines the previous developmental stage and by that enable developmental progression. Further research has shown that miRNAs provide a strong regulatory network which allows cells to quickly transition from one stage to the other, as well as to allow the whole organism to change course during embryonic development, adulthood and aging (Carthew et al., 2016, Garg and Cohen, 2014). miRNAs can regulate many types of transitions, such as stem cells to differentiated progeny, dynamic adherence between cells or internal regulation of the cell cycle (Ma et al., 2010, Melton et al., 2010, Yu et al., 2009). However, miRNAs also dictate stable cellular properties like fate commitment or protection from apoptosis. In this latter role, antiapoptotic miRNAs act as survival factors by blocking the expression of proapoptotic genes and provide a unique defense mechanism from apoptotic events (Brennecke et

Abbreviations used in this paper: miRNA, microRNA. *Address correspondence to: HilaToledano. Department of Human Biology, Faculty of Natural Sciences, University of Haifa, 199 Aba-Hushi Avenue, Mount Carmel,
Haifa, 3498838, Israel. Tel: +972-4-8280754. E-mail: hila@ @sci.haifa.ac.il - (iD) http://orcid.org/0000-0001-9409-7728

Accepted: 10 August 2016.

ISSN: Online 1696-3547, Print 0214-6282 
al., 2003, Carthew et al., 2016).

A miRNA regulates its $m R N A$ targets through binding to a small primary recognition sequence of $6-8$ nucleotides. These regulatory sequences are located throughout the mRNA but their presence is predominantly in the $3^{\prime}$ untranslated region (3'UTR). The 6 nucleotides seed sequence is located at the 5' of the miRNA, between nucleotides 2-8. This seed recognizes and base-pair with the anti-sense recognition sequence of the mRNA (Ghildiyal and Zamore, 2009). Because this type of recognition is obligatory, mRNA targets for each miRNA can be bioinformatically predicted. Indeed several hundreds to thousands mRNAs are predicted targets for each miRNA (Shin et al., 2010). miRNA target prediction also takes into account the free energy of miRNA and mRNA interactions and $m R N A$ recognition site conservation throughout evolution (Enright et al., 2003, Lewis et al., 2005). While some targets were experimentally confirmed, most of the predicted targets are yet to be validated. The use of bioinformatics often leads to false-positive identification, as experimentally proven using a shuffled miRNA sequence (Lewis et al., 2003). Therefore, not every mRNA that contains the seed sequence is indeed a direct target of the corresponding miRNA. Furthermore, even when a mRNA is a possible target for a certain miRNA, both should be adequately expressed in the same cell at the same time for repression to occur, which is not always the case.

Some studies using molecular biology techniques suggest that a single miRNA downregulates many mRNA targets simultaneously. This was shown in mammalian models where, for example, mir-1 and mir-124 that are mainly expressed in the muscle and brain were found to downregulate 96 and 174 genes, respectively. However, these experiments were done by over-expression of the selected miRNA in cells that do not normally express such high levels (Lim et al., 2005).

Therefore, one of the general questions of miRNA biology is whether in a given spatial and temporal state, a miRNA targets many mRNAs, as may be suggested by computational and molecular biology studies, or rather represses a single crucial target. Current genetic studies in Drosophila melanogaster reveal that individual targets/pathways are often major effectors of miRNAmediated phenotypes (Bejarano et al., 2012). In this review we will highlight the unique advantages of miRNA research in Drosophila and present selected examples to emphasize the strength of model organisms such as Drosophila to help distinguish whether "functional targeting" of individual targets are rare or actually common.

\section{The unique toolbox of Drosophila for miRNA research}

About 140 miRNAs were annotated in Drosophila with high confidence, from which 78 display $\geq 70 \%$ sequence similarity to human orthologous (Kozomara and Griffiths-Jones, 2011). Drosophila offers several unique advantages in the study of miRNAs as detailed herein.

The biggest advantage is the availability of genomewide collections of inducible loss of function (LOF) and gain of function (GOF) individual miRNAs (Bejarano et al., 2012, Fulga etal., 2015, Schertel et al., 2012). Three of these recently generated collections include Upstream Activating Sequence (UAS) to enable cell specific GOF or LOF of annotated miRNAs in desired tissues with the appropriate GAL4 drivers. The bipartite UAS-GAL4 system enables selective spatial expression of miRNAs from the above collections whereas

\section{A Control-sensor:}
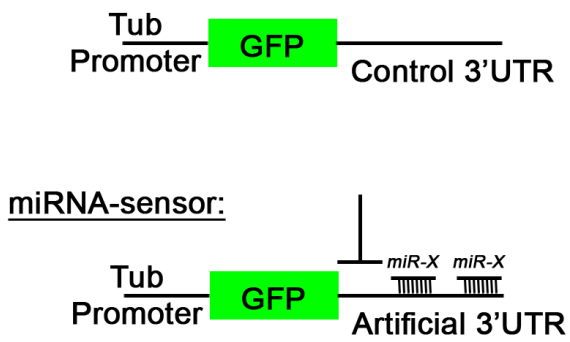

\section{B miRNA-sponge:}

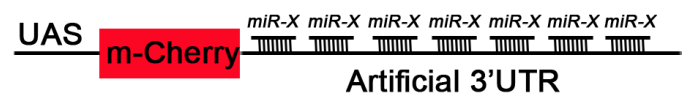

Fig. 1. Schematic representation of miRNA-sensor, control-sensor and miRNA-sponge. (A) miRNA- and control-sensors are driven by a ubiquitous promoter (e.g. Tubulin) and contain a reporter (e.g. GFP) coding region. The miRNA-sensor contains an artificial 3'UTR with 2-3 repeats of antisense sequences towards a given miRNA (mir-X), whereas the control-sensor contains a control 3'UTR (Brennecke et al., 2003, Schertel et al., 2012). (B). An miRNA-sponge contains a UAS element to drive spatial restricted expression with an appropriate GAL4 driver, followed by a reporter (e.g. $m$-Cherry) coding region. An artificial 3'UTR containing many repeats of antisense sequences towards a given miRNA (mir-X) is used as a decoy to deplete mir-X from a desired cell population (Fulga et al., 2015, Herranz et al., 2012).

otherwise overall loss or gain expression may be lethal (Phelps and Brand, 1998). Moreover, a temperature-sensitive allele of the GAL80 ${ }^{\text {ts }}$ repressor enables conditional expression restricted to a certain developmental stage or during adulthood thus providing spatially and temporally restricted expression (Suster et al., 2004).

The LOF sponge collection includes UAS-mCherry-miRNAsponge transgenes that permit conditional miRNA inactivation in specific cells (Fig. 1B). Each sponge transgene includes an artificial 3'UTR region following mCherry coding sequence. The 3'UTR region contains 20 binding sites for a given miRNA that are antisense sequences that act as a decoy to dilute miRNA expression (Ebert and Sharp, 2010, Fulga et al., 2015). The collection also includes mCherry to validate transgene expression.

The two GOF miRNA transgene collections that were recently generated cover the entire repertoire of Drosophila miRNAs and offer a useful tool to complement the LOF approaches (Bejarano et al., 2012, Schertel et al., 2012). Inducible GOF expression also circumvents redundancy difficulties of miRNAs for which LOF conditions do not yield a distinct phenotype. In one of the GOF collections, the transgenes are marked by DsRed to enable in-vivo tracking of miRNA overexpression (Bejarano et al., 2012). The joint effect of the GOF phenotype and its LOF counterpart, along with their coverage of each individual miRNA enables their use for genetic screens, individual miRNA research and identification of synergies between signaling pathways and miRNAs. So far these collections revealed hundreds of distinct phenotypes illustrating the power of genetics in identifying potent and unexpected functions of miRNAs that cannot be predicted by computational approaches and 
molecular biology studies (Bejarano et al., 2012, Fulga et al., 2015, Schertel et al., 2012). Drosophila is currently the only species for which such collections of inducible miRNA transgenic tools exist.

In addition to the inducible collections, genomewide null-miRNA mutations were obtained by targeted approaches, collectively deleting $99 \%$ of Drosophila miRNAs (Chen et al., 2014). A similar genomewide collection was previously obtained in $C$. elegans in which mutations in the majority of miRNAs did not result in loss of viability or abnormal developmental phenotypes, probably due to functional redundancy (Miska et al., 2007). However, when tested in genetically sensitized backgrounds, $\sim 80 \%$ of $C$. elegans miRNAs showed mutant phenotypes, supporting the notion that redundant function exists to ensure robustness of developmental processes (Brenner et al., 2010). In contrast to the C. elegans null collection, $80 \%$ of Drosophila miRNA null mutants exhibited significant phenotypes in normal background, at least in one of the following assays: survival, fertility, lifespan, primordial germ cell (PGC) number, external morphology (e.g. eye or wing defects), climbing behavior or hemolymph-brain barrier (Chen et al., 2014, Miska et al., 2007). This makes Drosophila a preferable model and suggests that the null collection will be invaluable for any additional in vivo functional analysis of miRNAs (Chen et al., 2014, Miska et al., 2007).

Another important method that is often used for miRNA research in Drosophila is the miRNA-sensor (Fig. 1A). As miRNAs are not immunogenic and antibodies cannot be created and used to identify their expression, miRNA-sensors were developed to in vivo monitor the expression pattern of a given miRNA. Similarly to the previously described miRNA-sponges, miRNA-sensors use the ability of miRNAs to silence the expression of their specific targets for in-vivo detection. A miRNA-sensor is comprised of few of the complementary sequences of the selected miRNA (usually twothree repeats) within an artificial 3'UTR region of a reporter GFP sequence. If a certain miRNA is present in a given cell population, it activates the silencing mechanism leading to disappearance of the GFP signal. Expression of the transgene is driven by a ubiquitous promoter (e.g. Tubulin) and the GFP levels are compared to those observed in unaffected cells. A control sensor carrying a control 3'UTR should be tested as well to verify that the pattern of GFP expression does not merely reflect the pattern of the driver expression (Fig. 1). Furthermore, testing the sensors in respective mutants should reveal their reliability. Currently, sensors exist for only a small fraction of Drosophila miRNAs but creating a GFP sensor transgene to characterize a given miRNA expression and test for direct genetic interactions in vivo is relatively simple to perform (Brennecke et al., 2003, Schertel et al., 2012).

The last advantage we would like to review here is the existence of two Dicer RNase III enzymes in Drosophila, Dicer-1 and Dicer-2. Dicer is a key processor of small RNAs that acts at the cytoplasm during the final stages of their biogenesis (Ghildiyal and Zamore, 2009). In contrast to other model organisms (e.g. C. elegans and mice) that have one Dicer enzyme to process both miRNAs and small interfering RNAs (siRNAs), in Drosophila Dicer-1 is devoted to miRNA processing whereas Dicer-2 generates siRNAs (Lee et al., 2004, Miyoshi et al., 2010). This segregation enabled further identification of components that selectively regulate miRNA biogenesis (Siomi and Siomi, 2010). Moreover the segregation generates a unique opportunity to use to RNAi machinery as a tool to reduce Dicer-1 in a desired cell population and thus to remove
miRNA production in these cells only. This approach can be used in post-mitotic adult tissues as Dicer-1 null mutants are lethal during embryonic development and germline clones are not possible in non-dividing cells. Removing miRNA production selectively from a group of cells in vivo may provide a means to identify critical and unique functions of miRNAs.

\section{DrosophilamiRNAs downregulate distinct major targets}

In this part we focus on a small fraction of the miRNA research done in Drosophila using the unique tools described above. All of these examples prove that at a certain place and time a single mRNA target is responsible for a unique miRNA phenotype (Fig. 2).

bantam (ban) is one of the most critical miRNAs for Drosophila development and its null mutants exhibit significantly reduce viability (Brennecke et al., 2003, Chen et al., 2014). GOF studies have shown that ban induces cell growth and a GFP-ban sensor revealed a striking correlation between its expression pattern and cell proliferation (Brennecke et al., 2003). Moreover, a ban-sponge used to deplete ban expression in selected dorsal compartment of the wing imaginal disc resulted in reduced tissue growth (Herranz et al., 2012). Co-expression of the ban-sensor and the ban sponge led to higher levels of sensor GFP levels proving the efficiency of the ban-sponge in depleting ban in vivo (Fig. 1). Consistent with its role in cell growth, ban is transcriptionally activated downstream of the Hippo-Yorkie, BMP and EGFR growth control signaling pathways that determine cell proliferation and organ size (Herranz et al., 2012, Nolo et al., 2006, Oh and Irvine, 2011, Thompson and Cohen, 2006). ban positively affects tissue growth by suppressing negative growth regulators, one being the pro-apoptotic IAP antagonist, Hid (Chen et al., 2014). The mRNA of the proapoptotic hid possesses five recognition elements for ban seed in its 3'UTR and hid was characterized as a bona-fide target for ban (Brennecke et al., 2003). Thus, bancan provide the cells protection from apoptosis and enable cell proliferation during development because it acts downstream of many apoptotic signals at the execution level of Hid expression. In the ovary of adult females ban provides selective protection from apoptosis only to the germline stem cell (GSC) population by blocking translation of the same target, hid. Upon apoptosis induction by genotoxic signals such as irradiation, the stem cells remain intact while the neighboring differentiated cells undergo apoptosis (Xing et al., 2015). Although there is no ban orthologue in mammals, these studies paved the way for identification of antiapoptotic miRNAs and their role in human diseases including cancer (Lima et al., 2011). ban is also expressed in another stem cell population, the larval brain neuroblasts while it is absent from differentiated progeny as revealed by ban GFP-sensor. In neuroblasts ban downregulates the differentiation factors prospero and brat to enable stem cell maintenance (Weng and Cohen, 2015). Another critical cellular function of ban is to regulate the circadian rhythm. Circadian clocks act autonomously in all cells and are generated by feedback loops of interconnected transcription factors including Clock that is present in limiting amounts to ensure adaptation to daily environmental cycles. GOF of clock that carries mutations in the recognition elements for ban and is thus resilient for post-transcriptional regulation, resulted in defects in circadian neurons development (Lerner et al., 2015). These examples reveal that ban miRNA has diverse functions in different time, cellular and tissue contexts, each mediated by downregulation of one 
key mRNA target.

Another multifunctional miRNA in Drosophila is miR-9a. Unlike ban, the miR-9a nucleotide sequence is evolutionarily conserved between Drosophila, mice and humans. Moreover, miR-9amutants are viable, fertile and reach adulthood (Li et al., 2006). miR-9a is a clear example whereby an easily distinguished LOF morphological phenotype can be mimicked by manipulating the recognition site of a single mRNA target of the Drosophila LIM-Only (dLMO) gene. dLMO serves as a transcription cofactor which inhibits Apterous directly (Milan et al., 1998). Apterous is required for the integrity of the Drosophila wings on the dorsal side. When miR-9a was deleted from the genome, a unique no-margin wing phenotype occurred. A similar phenotype was obtained in a separate experiment in which the 3'UTR of $d L M O$ was removed. These two identical phenotypes that emerged inconsequently to each other suggest that there is a strong and exclusive link between miR-9a and its target, $d L M O$ (Biryukova et al., 2009). This phenotype was a result of apoptosis, and was completely reversed when a single allele of $d L M O$ was removed, thus inhibiting its endogenous expression, regardless of the presence of miR-9a. Moreover, GOF of UAS-DsRed:miR-9a in the pouch domain of the wing imaginal disc resulted in specific reduction of GFP-dLMO sensor, implying a direct regulation of miR-9a via dLMO 3'UTR (Bejarano et al., 2010). The miR-9a recognition element is conserved in the human ortholouge of the T-cell acute leukemia oncogene (LMO2), suggesting that miR-9 may also downregulates LMO2 expression under harmful levels (Biryukova et al., 2009).

miR-9a mutants exhibit additional morphological phenotypes: ectopic formation of sensory neurons in embryos and excessive number of sensory organs in adults. miR-9a is expressed in epithelial cells prior to their specification as SOP and downregulates the expression of the transcription factor senseless thereby

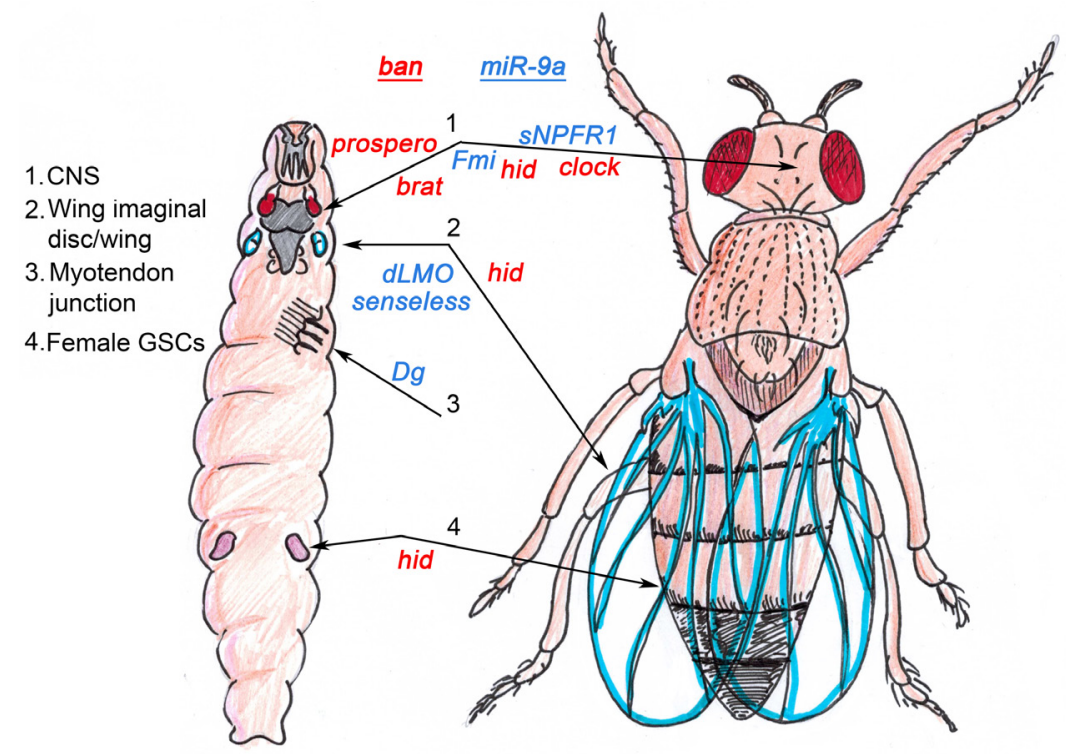

Fig. 2. ban and miR-9a downregulate direct individual targets in distinct developmental stages and cells. ban (red) regulates hid (red) in the wing imaginal disc, brain hemisphere and adult female GSCs. ban also regulates clock (red) in the brain, prospero (red) and brat (red) in neuroblasts. miR-9a (blue) regulates SNPFR1 in adult brain and Fmi in larvae CNS. miR-9a also regulates dLMO and senseless in the wing imaginal disc and $\mathrm{Dg}$ in the tendons. inhibiting the SOP fate in the surrounding epithelial cells and limit the number of SOP (Carthew et al., 2016, Li et al., 2006). The interaction between $\mathrm{miR}-9 \mathrm{a}$ and its target senseless also helps to ensure developmental robustness during SOP specification as miR-9a mutants show high variability among individuals in the number of sensory organs. This variability increases when miR-9a mutants are exposed to environmental and/or genetic variations (Cassidy et al., 2013).

miR-9a has another role in a different time and place. During embryonic development, it regulates the expression of Dystroglycan $(\mathrm{Dg})$, an extracellular matrix (ECM) receptor necessary for the formation of myo-tendious junctions. Muscles express Dg while their neighboring tendons express miR-9a which down regulates Dg. This allows correct formation of muscle-tendon connections. miR-9a serves as a "bouncer" selectively blocking expression of muscle factors in tendons and thus protecting their specific cell fate. (Yatsenko \& Shcherbata, 2014).

In the Drosophila class I dendrite arborization (da) neurons, miR-9a attenuates dendrite development by suppressing the cadherin protein Flamingo (Fmi). miR-9a lacks from the neurons and expresses exclusively in the epithelium thus the expression of epithelial but not neuronal Fmi is downregulated. Fmi forms homophilic adhesion interactions and influences axonal growth and navigation by affecting the adhesion between primary and following axons. In dendrite growth, Fmi plays a repressive role and in the absence of miR-9a, the excess Fmi binds to neuronal Fmi and causes unnecessary dendrite growth (Wang et al., 2016). Interestingly, in mammals miR-9 also regulates adhesion by downregulating another cadherin molecule, Epithelial-cadherin. miR-9a is upregulated in breast cancer leading to increased cell motility and invasiveness (Ma et al., 2010).

Another proven target of $m i R-9 a$ is the short neuropeptide receptor F1 (sNPFR1), a modulator of insulin signaling. The Insulin/IGF pathway regulates body growth in Drosophila, through the neurosecratory insulin-producing cells located in the fly brain. The cells produce three insulin-like peptides (Dilps) among which, Dilp2 is the dominant one controlling body growth. Dilp2 expression is regulated by the connection between SNPF and its receptor, sNPFR1. When overexpressed in the insulin-producing cells of the brain, miR-9a inhibits the expression of sNPFR1, which in turn leads to reduced body size, wing length and pupal volume. When miR $9 a$ is knocked-down, the phenotype is reversed to an increased body size and wing length, as well as larger pupal volume (Suh et al., 2015).

miR-9a mutant flies also have an aging phenotype of a short lifespan. It will be interesting to identify the mRNA targets whose downregulation is essential for a normal lifespan (Chen et al., 2014).Three additional miRNAs where found to regulate aging related phenotypes: let-7, miR-34 and miR-1000 (Liu et al., 2012, Toledano et al., 2012, Verma et al., 2015). let-7 was initially described as a regulator of the developmental timing pathway, namely the heterochronic pathway (Ambros, 2011). let-7 regulation of stem cells during aging was first described for neuronal stem cells in the mouse forebrain, expending the heterochronicfunction towards adulthood and aging (Nishino et al., 2008, 
Toledano, 2013). In the Drosphila male (germline stem cell) GSC niche, let-7 is expressed in the hub cells that represent a major component of the somatic niche. We demonstrated that elevated levels of let-7in the hub of aged males initiate a cascade of events that culminates in decreased niche function and GSCs loss. The increase in the levels of let-7 directly regulates the mRNA of the evolutionarily conserved RNA binding protein imp, and reduces self-renewal function of the niche (Toledano et al., 2012).

miR-34 and miR-1000 deficient animals have a shortened lifespan and they exhibit early-onset neurodegeneration (Liu et al., 2012, Verma et al., 2015). Furthermore, they both exhibit brain-enriched and age-modulated characteristics. However, while the levels of $m i R-34$ increase in the brain during aging, the levels of $m i R-1000$ decreases. Eip74EF (E74A) was characterized as a miR-34 target and elevated levels of E74A were found in the miR-34 mutant to be responsible for the degeneration and short lifespan (Liu et al., 2012).

miR-1000 is expressed in the Drosophila CNS throughout the life of the fly, its target being the vesicular glutamate transporter (VGlut). VGlut is responsible for loading glutamate onto synaptic vesicles to be released at the synaptic junction. When miR-1000 was deleted from the genome, there was an overexpression of VGlut which resulted in excessive glutamate release leading to fly death due to excessive stimulation of nerve cells. When rescued through reintroduction of miR-1000, the flies survived at a rate similar to the control (Verma et al., 2015). This shows a tight connection between one miRNA and one mRNA target, in a specific tissue that affect the overall organism lifespan.

\section{Concluding remarks}

Our knowledge of stages in animal development relies on tools and techniques designed to detect cellular events that occur within the whole organism. In the past, Drosophila was mainly used to perform forward- and reverse genetic screens. Currently, the development of advanced biochemical and molecular biology techniques has enabled the use of Drosophila to manipulate gene expression in small specialized cell populations. In the miRNA field, Drosophila became an attractive model to study dynamic interplays between miRNA and mRNA targets. It enables us to specifically alter one small binding sequence of miRNA-mRNA and to study the effect at multiple levels of cellular and whole organism function. In recent years, miRNA research in Drosophila largely contributed to the understanding that this class of posttranscriptional regulators has a major role in gene expression throughout all cycles of the lifespan.

In this review, we discuss the use of unique tools established for miRNA research in Drosophila and show that miRNA at a certain cellular and temporal event regulate only one key target (Fig. 2). This perspective counteracts computational approaches that yielded hundreds to thousands direct target predictions per a single animal miRNA. These predictions were supported by systematic profiling of the transcriptome and suggested that miRNAs have a broad and subtle "fine-tuning" type of regulation.

miRNA research in Drosophila emphasizes the gap between bioinformatics and phenotype characterization of GOF and LOF transgenes in model organism. However, at this stage we cannot rule this notion on higher organisms and further research in mammals will confirm whether we can generalize that at the right place and time miRNAs regulate single crucial targets in all animals.

\section{References}

AMBROS, V. (2011). MicroRNAs and developmental timing. Curr Opin Genet Dev 21: $511-517$

BEJARANO, F., BORTOLAMIOL-BECET, D., DAI, Q., SUN, K., SAJ, A., CHOU, Y.T., RALEIGH, D.R., KIM, K., NI, J.Q., DUAN, H. et al., (2012). A genome-wide transgenic resource for conditional expression of Drosophila microRNAs. Development 139: 2821-2831.

BEJARANO, F., SMIBERT, P. and LAI, E.C. (2010). miR-9a prevents apoptosis during wing development by repressing Drosophila LIM-only. Dev Biol 338: 63-73.

BIRYUKOVA, I., ASMAR, J., ABDESSELEM, H. and HEITZLER, P. (2009). Drosophila mir-9a regulates wing development via fine-tuning expression of the LIM only factor, dLMO. Dev Biol 327: 487-496.

BRENNECKE, J., HIPFNER, D.R., STARK, A., RUSSELL, R.B. and COHEN, S.M. (2003). bantam encodes a developmentally regulated microRNA that controls cell proliferation and regulates the proapoptotic gene hid in Drosophila. Cell113:25-36.

BRENNER, J.L., JASIEWICZ, K.L., FAHLEY, A.F., KEMP, B.J. and ABBOTT, A.L. (2010). Loss of individual microRNAs causes mutant phenotypes in sensitized genetic backgrounds in C. elegans. Curr Biol 20: 1321-1325.

CARTHEW, R. W., AGBU, P. and GIRI, R. (2016). MicroRNA function in Drosophila melanogaster. In Seminars in cell \& developmental biology. Academic Press.

CASSIDY, J.J., JHA, A.R., POSADAS, D.M., GIRI, R., VENKEN, K.J., JI, J., JIANG, H., BELLEN, H.J., WHITE, K.P. and CARTHEW, R.W. (2013). miR-9a minimizes the phenotypic impact of genomic diversity by buffering a transcription factor. Cell 155: 1556-1567.

CHEN, Y.W., SONG, S., WENG, R., VERMA, P., KUGLER, J.M., BUESCHER, M., ROUAM, S. and COHEN, S.M. (2014). Systematic study of Drosophila microRNA functions using a collection of targeted knockout mutations. Dev Cell31: 784-800.

EBERT, M.S. and SHARP, P.A. (2010). MicroRNA sponges: progress and possibilities. RNA 16: 2043-2050.

ENRIGHT, A.J., JOHN, B., GAUL, U., TUSCHL, T., SANDER, C. and MARKS, D.S. (2003). MicroRNA targets in Drosophila. Genome Biol 5: R1.

FULGA, T.A., MCNEILL, E.M., BINARI, R., YELICK, J., BLANCHE, A., BOOKER, M., STEINKRAUS, B.R., SCHNALL-LEVIN, M., ZHAO, Y., DELUCA, T. et al., (2015). A transgenic resource for conditional competitive inhibition of conserved Drosophila microRNAs. Nat Commun 6: 7279.

GARG, D. and COHEN, S.M. (2014). miRNAs and aging: a genetic perspective. Ageing Res Rev 17: 3-8.

GHILDIYAL, M. and ZAMORE, P.D. (2009). Small silencing RNAs: an expanding universe. Nat Rev Genet 10: 94-108.

HERRANZ, H., HONG, X. and COHEN, S.M. (2012). Mutual repression by bantam miRNA and Capicua links the EGFR/MAPK and Hippo pathways in growth control. Curr Biol 22: 651-657.

KOZOMARA, A. and GRIFFITHS-JONES, S. (2011). miRBase: integrating microRNA annotation and deep-sequencing data. Nucleic Acids Res 39: D152-D157.

LEE, R.C. and AMBROS, V. (2001). An extensive class of small RNAs in Caenorhabditis elegans. Science 294: 862-864.

LEE, R.C., FEINBAUM, R.L. and AMBROS, V. (1993). The C. elegans heterochronic gene lin-4 encodes small RNAs with antisense complementarity to lin-14. Cell 75: 843-854.

LEE, Y.S., NAKAHARA, K., PHAM, J.W., KIM, K., HE, Z., SONTHEIMER, E.J. and CARTHEW, R.W. (2004). Distinct roles for Drosophila Dicer-1 and Dicer-2 in the siRNA/miRNA silencing pathways. Cell 117: 69-81.

LERNER, I., BARTOK, O., WOLFSON, V., MENET, J.S., WEISSBEIN, U., AFIK, S., HAIMOVICH, D., GAFNI, C., FRIEDMAN, N., ROSBASH, M. et al., (2015). Clk post-transcriptional control denoises circadian transcription both temporally and spatially. Nat Commun 6: 7056.

LEWIS, B.P., BURGE, C.B. and BARTEL, D.P. (2005). Conserved seed pairing, often flanked by adenosines, indicates that thousands of human genes are microRNA targets. Cell 120: 15-20.

LEWIS, B.P., SHIH, I.H., JONES-RHOADES, M.W., BARTEL, D.P. and BURGE, C.B. (2003). Prediction of mammalian microRNA targets. Cell 115: 787-798. 
LI, Y., WANG, F., LEE, J.A. and GAO, F.B. (2006). MicroRNA-9a ensures the precise specification of sensory organ precursors in Drosophila. Genes Dev20:2793-2805.

LIM, L.P., LAU, N.C., GARRETT-ENGELE, P., GRIMSON, A., SCHELTER, J.M., CASTLE, J., BARTEL, D.P., LINSLEY, P.S. and JOHNSON, J.M. (2005). Microarray analysis shows that some microRNAs downregulate large numbers of target mRNAs. Nature 433: 769-773.

LIMA, R.T., BUSACCA, S., ALMEIDA, G.M., GAUDINO, G., FENNELL, D.A. and VASCONCELOS, M.H. (2011). MicroRNA regulation of core apoptosis pathways in cancer. Eur J Cancer 47: 163-174.

LIU, N., LANDREH, M., CAO, K., ABE, M., HENDRIKS, G.J., KENNERDELL, J.R., ZHU, Y., WANG, L.S. and BONINI, N.M. (2012). The microRNA miR-34 modulates ageing and neurodegeneration in Drosophila. Nature 482: 519-523.

MA, L., YOUNG, J., PRABHALA, H., PAN, E., MESTDAGH, P., MUTH, D., TERUYAFELDSTEIN, J., REINHARDT, F., ONDER, T.T., VALASTYAN, S. et al., (2010). miR-9, a MYC/MYCN-activated microRNA, regulates E-cadherin and cancer metastasis. Nat Cell Biol 12: 247-256.

MELTON, C., JUDSON, R.L. and BLELLOCH, R. (2010). Opposing microRNA families regulate self-renewal in mouse embryonic stem cells. Nature 463: 621-626.

MILAN, M., DIAZ-BENJUMEA, F.J. and COHEN, S.M. (1998). Beadex encodes an LMO protein that regulates Apterous LIM-homeodomain activity in Drosophila wing development: a model for LMO oncogene function. Genes Dev 12: 2912-2920.

MISKA, E.A., ALVAREZ-SAAVEDRA, E., ABBOTT, A.L., LAU, N.C., HELLMAN, A.B., MCGONAGLE, S.M., BARTEL, D.P., AMBROS, V.R. and HORVITZ, H.R. (2007). Most Caenorhabditis elegans microRNAs are individually not essential for development or viability. PLoS Genet 3: e215.

MIYOSHI, K., MIYOSHI, T., HARTIG, J.V., SIOMI, H. and SIOMI, M.C. (2010). Molecular mechanisms that funnel RNA precursors into endogenous small-interfering RNA and microRNA biogenesis pathways in Drosophila. RNA 16: 506-515.

NISHINO, J., KIM, I., CHADA, K. and MORRISON, S.J. (2008). Hmga2 promotes neural stem cell self-renewal in young but not old mice by reducing p16Ink4a and p19Arf Expression. Cell 135: 227-239.

NOLO, R., MORRISON, C.M., TAO, C., ZHANG, X. and HALDER, G. (2006). The bantam microRNA is a target of the hippo tumor-suppressor pathway. Curr Biol 16: 1895-1904.

$\mathrm{OH}, \mathrm{H}$. and IRVINE, K.D. (2011). Cooperative regulation of growth by Yorkie and Mad through bantam. Dev Cell 20: 109-122.

PHELPS, C.B. and BRAND, A.H. (1998). Ectopic gene expression in Drosophila using GAL4 system. Methods 14: 367-379.

SCHERTEL, C., RUTISHAUSER, T., FORSTEMANN, K. and BASLER, K. (2012). Functional characterization of Drosophila microRNAs by a novel in vivo library. Genetics 192: 1543-1552.
SHIN, C., NAM, J.W., FARH, K.K., CHIANG, H.R., SHKUMATAVA, A. and BARTEL, D.P. (2010). Expanding the microRNAtargeting code: functional sites with centered pairing. Mol Cell 38: 789-802.

SIOMI, H. and SIOMI, M.C. (2010). Posttranscriptional regulation of microRNA biogenesis in animals. Mol Cell 38: 323-332.

SUH, Y.S., BHAT, S., HONG, S.H., SHIN, M., BAHK, S., CHO, K.S., KIM, S.W., LEE, K.S., KIM, Y.J., JONES, W.D. et al., (2015). Genome-wide microRNA screening reveals that the evolutionary conserved miR-9a regulates body growth by targeting SNPFR1/NPYR. Nat Commun 6: 7693

SUSTER, M.L., SEUGNET, L., BATE, M. and SOKOLOWSKI, M.B. (2004). Refining GAL4-driven transgene expression in Drosophila with a GAL80 enhancer-trap. Genesis 39: 240-245.

THOMPSON, B.J. and COHEN, S.M. (2006). The Hippo pathway regulates the bantam microRNA to control cell proliferation and apoptosis in Drosophila. Cell 126: $767-774$

TOLEDANO, H. (2013). The role of the heterochronic microRNA let-7 in the progression of aging. Exp Gerontol 48: 667-670.

TOLEDANO, H., D'ALTERIO, C., CZECH, B., LEVINE, E. and JONES, D.L. (2012) The let-7-Imp axis regulates ageing of the Drosophila testis stem-cell niche. Nature 485: 605-610.

VERMA, P., AUGUSTINE, G.J., AMMAR, M.R., TASHIRO, A. and COHEN, S.M. (2015) A neuroprotective role for microRNA miR-1000 mediated by limiting glutamate excitotoxicity. Nat Neurosci 18: 379-385.

WANG, Y., WANG, H., LI, X. and LI, Y. (2016). Epithelial microRNA-9a regulates dendrite growth through Fmi-Gq signaling in Drosophila sensory neurons. Dev Neurobiol 76: 225-237.

WENG, R. and COHEN, S.M. (2015). Control of Drosophila Type I and Type II central brain neuroblast proliferation by bantam microRNA. Development 142:3713-3720.

WIGHTMAN, B., HA, I. and RUVKUN, G. (1993). Posttranscriptional regulation of the heterochronic gene lin-14 by lin-4 mediates temporal pattern formation in $\mathrm{C}$ elegans. Cell 75: 855-862

XING, Y., SU, T.T. and RUOHOLA-BAKER, H. (2015). Tie-mediated signal from apoptotic cells protects stem cells in Drosophila melanogaster. Nat Commun 6: 7058

YATSENKO, A. S., and SHCHERBATA, H. R. (2014). Drosophila miR-9a targets the ECM receptor Dystroglycan to canalize myotendinous junction formation. Dev. Cell, 28: 335-348.

YU, J.Y., REYNOLDS, S.H., HATFIELD, S.D., SHCHERBATA, H.R., FISCHER, K.A WARD, E.J., LONG, D., DING, Y. and RUOHOLA-BAKER, H. (2009). Dicer1-dependent Dacapo suppression acts downstream of Insulin receptor in regulating cell division of Drosophila germline stem cells. Development 136: 1497-1507. 


\section{Further Related Reading, published previously in the Int. J. Dev. Biol.}

Small RNA pathways in Schmidtea mediterranea

Alissa M. Resch and Dasaradhi Palakodeti

Int. J. Dev. Biol. (2012) 56: 67-74

https://doi.org/10.1387/ijdb.113436ar

Spatially controlled expression of the Drosophila pseudouridine synthase RluA-1 Chung-Chi Wang, Jun-Chih Lo, Cheng-Ting Chien and Min-Lang Huang

Int. J. Dev. Biol. (2011) 55: 223-227

https://doi.org/10.1387/ijdb.103112cw

\section{Recent advances in Drosophila stem cell biology}

John Pearson, Lourdes López-Onieva, Patricia Rojas-Ríos and Acaimo González-Reyes Int. J. Dev. Biol. (2009) 53: 1329-1339

https://doi.org/10.1387/ijdb.072431.jp

Diverse miRNA spatial expression patterns suggest important roles in homeostasis and regeneration in planarians

Cristina González-Estévez, Varvara Arseni, Roshana S. Thambyrajah, Daniel A. Felix and A. Aziz Aboobaker

Int. J. Dev. Biol. (2009) 53: 493-50

https://doi.org/10.1387/ijdb.082825cg

\section{Plant microRNAs and development}

Sara Jover-Gil, Héctor Candela and María-Rosa Ponce

Int. J. Dev. Biol. (2005) 49: 733-744

https://doi.org/10.1387/ijdb.052015sj

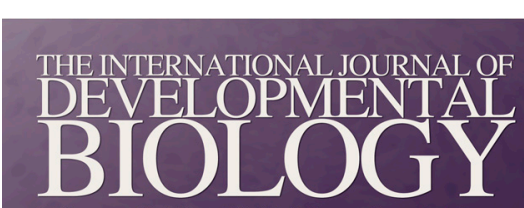

Volume 60 Nos. 10/11/12

Special Issue

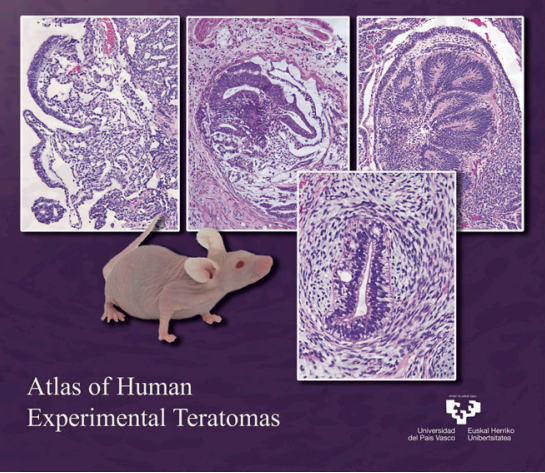

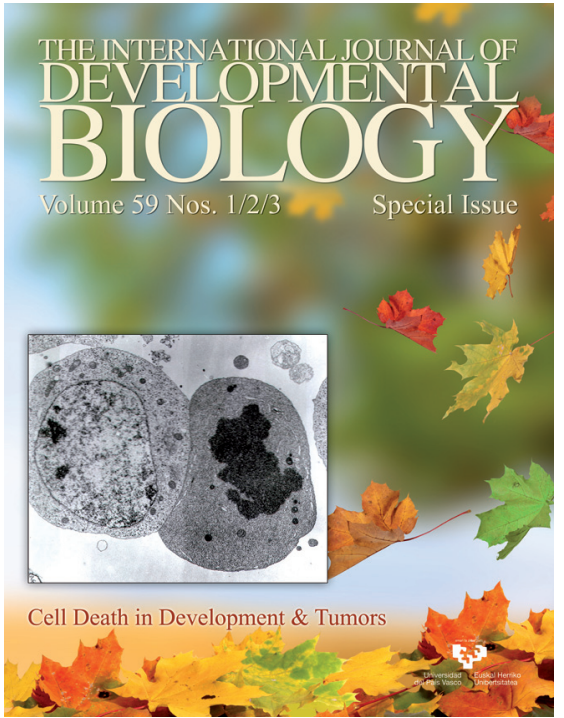
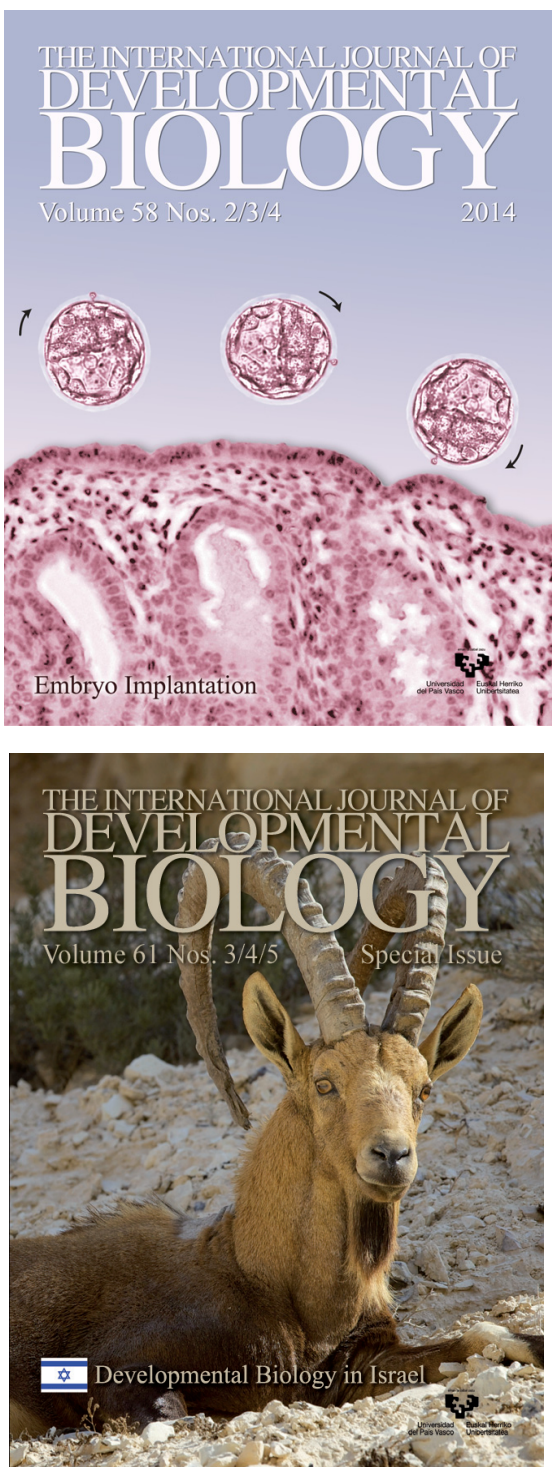